\title{
Respostas comportamentais apresentadas por longevos após internação na Unidade de Terapia Intensiva e retorno domiciliar
}

Behavioral responses presented by elderly people after hospitalization in the intensive care unit and return home

\section{Respuestas comportamentales presentadas por longevos después de la hospitalización en la Unidad de Cuidados Intensivos y regreso al hogar}

Jessica Lane Pereira Santos ${ }^{1}$ (D)

Larissa Chaves Pedreira ${ }^{1}$ (C)

Juliana Bezerra do Amaral ${ }^{1}$ (D)

Larissa Simões da Cruz Pessoa ${ }^{1}$ (D)

Elaine de Oliveira Souza² (D)

Aline Cristiane de Sousa

Azevedo Aguiar ${ }^{2}$ (D)

1. Universidade Federal da Bahia, Programa de Pós-Graduação em Enfermagem e Saúde. Salvador, BA, Brasil.

2. Universidade do Estado da Bahia Departamento de Educação. Guanambi, BA, Brasil.

\begin{abstract}
Resumo
Objetivo: identificar respostas comportamentais apresentadas por longevos no domicílio, após internação na Unidade de Terapia Intensiva e alta hospitalar. Método: estudo qualitativo, realizado com pessoas de 80 anos ou mais. 0 cenário da coleta de dados foi a Unidade de Terapia Intensiva e o domicílio de longevos que estiveram internados na unidade, por meio de entrevista aberta. Para análise dos dados, utilizou-se o método de análise de conteúdo de Bardin, pautada na teoria de Adaptação de Callista Roy. Todos os cuidados éticos foram respeitados. Resultados: emergiram-se duas categorias: Alterações do padrão de resposta às necessidades fisiológicas e perda de autonomia e Alterações psicossociais e comprometimento na realização de atividades cotidianas de lazer e laborais. Conclusão e implicações para a prática: as respostas comportamentais foram relacionadas aos modos fisiológicos e psicossociais, que culminaram em dependência e perda de autonomia para realização das atividades básicas de vida. Perceberam-se dificuldades vivenciadas no retorno ao domicílio, que provocaram alterações significativas na realização de atividades cotidianas, evidenciando a necessidade de se iniciar o preparo para o retorno domiciliar, pela equipe multiprofissional, ainda durante a hospitalização. Espera-se que estratégias visando melhorias dos resultados de cuidados críticos sejam implementadas nessa população.
\end{abstract}

Palavras-chave: Adaptação; Alta do Paciente; Idoso de 80 Anos ou mais; Resultados de Cuidados Críticos; Unidade de Terapia Intensiva.

\section{Abstract}

Objective: to identify the behavioral responses presented by elderly people at home after hospitalization at the intensive care unit and hospital discharge. Method: a qualitative study was conducted with people aged 80 years or older. The data collection setting was the intensive care unit and the homes of the elderly people hospitalized in the unit using open interviews. Data analysis consisted of Bardin's method of content analysis based on the Callista Roy adaptation model. All ethical aspects were respected. Results: two categories emerged: changes in the pattern of response to physiological needs and loss of autonomy and psychosocial changes and impairment in performing daily leisure and work activities. Conclusion and implications for practice: behavioral responses were related to physiological and psychosocial factors, culminating in dependence and loss of autonomy to perform basic life activities. Difficulties experienced in returning home were also noticed, which caused significan changes in daily activities, thereby evidencing the need for multidisciplinary teams to begin preparing for the return home during hospitalization. Strategies seeking to improve critical care outcomes are highly suggested to be implemented in this population.

Keywords: Adaptation; Patient Discharge; Aged, 80 and over; Critical Cares Outcomes; Intensive Care Units.

\section{RESUMEN}

Objetivo: identificar respuestas comportamentales presentadas por longevos en el domicilio después de internación en la Unidad de Cuidados Intensivos y alta hospitalaria. Método: estudio cualitativo, realizado con personas de 80 años o más. El escenario de la recolección de datos fue la Unidad de Cuidados Intensivos y el domicilio de longevos que estuvieron internados en la unidad por medio de entrevista abierta. Para el análisis de los datos se utilizó el método de análisis de contenido de Bardin, pautado en la teoría de Adaptación de Callista Roy. Todos los cuidados éticos fueron respetados. Resultados: surgieron dos categorías: Alteraciones del patrón de respuesta a las necesidades fisiológicas y pérdida de autonomía y Alteraciones psicosociales y compromiso en la realización de actividades cotidianas de ocio y laborales. Conclusión e implicaciones para la práctica: las respuestas comportamentales fueron relacionadas a los modos fisiológicos y psicosociales, que culminaron en dependencia y pérdida de autonomía en la realización de las actividades básicas de la vida. Se percibieron dificultades vividas en el retorno a domicilio, que provocaron alteraciones significativas en la realización de actividades cotidianas, revelando la necesidad de iniciar la preparación para el retorno domiciliar por el equipo multiprofesional aún durante la hospitalización. Se espera que estrategias que busquen mejoras de los resultados de cuidados críticos sean implementadas en esa población.

Palabras clave: Adaptación; Alta del Paciente; Anciano de 80 o más Años; Resultados de Cuidados Críticos; Unidades de Cuidados Intensivos.
Recebido em 02/07/2021.

Aprovado em 21/10/2021. 


\section{INTRODUÇÃO}

Como consequência do envelhecimento populacional, observa-se um aumento de longevos, definidos como pessoas de 80 anos ou mais. Esse crescente perfil exige novas demandas no cuidado, decorrentes do aumento das doenças, incapacidadese internamentos, sendo imperativo empreender a rápida adaptação dos serviços de saúde para o atendimento das necessidades dessa população'.

Estudos demonstram um aumento da hospitalização na população idosa, já que, em decorrência do envelhecimento populacional, tem-se elevação da incidência das doenças crônicas degenerativas, com período de agudização destas, promovendo, também, o internamento na Unidade de Terapia Intensiva (UTI) ${ }^{2}$.

A hospitalização da pessoa idosa desencadeia uma sucessão de eventos que, frequentemente, resultam na redução da capacidade funcional e da qualidade de vida, complicações que podem não se relacionar com o problema que levou à admissão hospitalar. Dessa forma, nem sempre a hospitalização resulta em melhora do quadro de saúde ${ }^{3}$.

Pesquisa realizada com 128 longevos internados nas unidades clínicas, cirúrgicas e na emergência do Hospital São Paulo, observou que a maioria apresentou grau máximo de dependência durante o processo de hospitalização, sendo verificado declínio do estado geral da saúde devido à limitação em realizar as Atividades de Vida Diária (AVD) e o desconhecimento dos profissionais de saúde em compreender a necessidade de estimulá-las ${ }^{4}$.

Outro estudo de revisão sistemática, que avaliou 48 pesquisas, identificou que os domínios mais afetados pela doença crítica foram os relacionados ao papel físico, à função física, vitalidade e função social. Entretanto, constatou-se que houve melhora na qualidade de vida referente a esses mesmos domínios, um ano após a alta, enfatizando que as intervenções auxiliares na recuperação, depois de uma doença crítica, são mais exitosas no primeiro ano após a alta hospitalar ${ }^{5}$.

Nesse sentido, a equipe de saúde da UTI deve realizar o adequado preparo do longevo e da sua família para o retorno ao domicílio, posteriormente à alta hospitalar, de modo a propiciar a promoção da saúde. Para tanto, é fundamental traçar metas individualizadas, a partir das necessidades observadas durante a internação hospitalar, que possibilitem a prevenção de agravos e a reabilitação. Com isso, espera-se que, no retorno domiciliar, o longevo tenha mais qualidade de vida e reduza a necessidade de (re)hospitalização6.

A Teoria da Adaptação de Callista Roy considera que o objetivo da enfermagem é promover a adaptação dos indivíduos e grupos nos quatro modos adaptativos: fisiológico, de autoconceito, interdependência e desempenho de papel 7 . De acordo com Roy e Andrews, "é o comportamento que demonstra a eficácia com que os mecanismos de resistência são capazes de se adaptar aos estímulos que afetam a pessoa", e as respostas apresentadas devem ser sistematicamente observadas em cada modo adaptável: ${ }^{8: 63}$.
Cabe ao profissional de enfermagem planejar e desenvolver estratégias para o cuidado que englobe ações nos diferentes níveis de atendimento à saúde, sendo a pessoa assistida até o retorno das suas atividades de vida diária e reinserção social ${ }^{7}$.

Diante das pontuações acima, questiona-se quais são as respostas comportamentais apresentadas por longevos no domicílio, após hospitalização na UTI. Objetiva-se identificar respostas comportamentais apresentadas por longevos no domicílio, após internação na Unidade de Terapia Intensiva e alta hospitalar.

A Teoria de Adaptação de Callista Roy fornecerá as nuances para compreender as respostas adaptativas observadas nos longevos, bem como possíveis alterações que vão além do campo fisiológico, identificadas quando de seu retorno ao domicílio. Tais alterações podem estar ligadas também aos campos psicológico, espiritual, social e/ou emocional, e requererão, do longevo, respostas adaptativas diante dos estímulos desencadeados pela hospitalização.

As respostas comportamentais apresentadas pelos longevos após a hospitalização denotam fatores que comprometem o retorno de atividades rotineiras em domicílio e interferem na sua autonomia. Ademais, mediante a identificação de respostas comportamentais desencadeadoras de uma adaptação ineficaz, tornar-se-á possível o planejamento de estratégias que busquem melhorias dos resultados de cuidados críticos, de modo a prevenir riscos associados à hospitalização, visando a promoção da qualidade de vida ao longevo no retorno domiciliar.

\section{MÉTODOS}

\section{Tipo de estudo}

Trata-se de um estudo descritivo, exploratório, com abordagem qualitativa.

\section{Local do estudo}

Pesquisa realizada no Nordeste do Brasil, em uma cidade localizada no Sudoeste do estado da Bahia.

O estudo foi realizado em dois locais: em um hospital público e na residência de longevos que estiveram internados na UTI do hospital em questão, após a alta hospitalar.

Essa UTI apresenta dez leitos destinados a pacientes adultos e idosos com demandas clínicas e cirúrgicas, localizada em um hospital geral que atende pacientes de variados municípios, inclusive de localidades rurais.

\section{Participantes da pesquisa}

A pesquisa foi desenvolvida com pessoas longevas que estiveram internadas na UTI e receberam alta hospitalar para o domicílio. Critérios de inclusão: pessoas de 80 anos ou mais que permaneceram internadas na unidade por um período mínimo de 24 horas, ainda que a sua alta hospitalar tenha sido proveniente de outra unidade; ter recebido alta hospitalar no período mínimo de uma semana e máximo de 12 meses; ter plenas condições para desenvolver um diálogo coerente na entrevista. 
Foram excluídos os longevos que, no momento da entrevista, não estavam em condições de saúde adequadas, apresentando, por exemplo, sonolência, hipoatividade, indisposição ou dor; ou em situações em que, após duas tentativas de contato telefônico para agendamento e/ou visita domiciliar, não foi alcançado o colaborador.

\section{Coleta de dados e instrumento da pesquisa}

A coleta de dados foi realizada em dois ambientes: na mencionada UTI, e na residência dos longevos que estiveram internados e receberam alta.

No cenário da UTI, para alcance das informações sobre os colaboradores da pesquisa, utilizou-se o livro de registros da unidade, que possibilitou identificar os idosos classificados como longevos, a sua data de internamento e de alta. Incluíram-se aqueles que estiveram internados e receberam alta da unidade no período de um ano, tendo, como data de base, o primeiro dia da visita dos pesquisadores, em 16 de outubro de 2017. Logo, a coleta ocorreu entre 16 de outubro de 2016 e 16 de outubro de 2017. Naquele momento, ainda foi realizada a busca do diagnóstico médico de internamento e a cidade de domicílio.

Após a primeira identificação no livro de registro, os pesquisadores utilizaram o livro de contatos da unidade para obterem o endereço de residência e o telefone de contato dos longevos identificados como elegíveis. Foi possível identificar 26 longevos; desses, 6 foram a óbito durante a internação e 20 tiveram alta da unidade. Por meio de contato telefônico, obteve-se a informação que cinco foram a óbito após retorno ao domicílio e dois apresentavam discurso incoerente, segundo informado pelo familiar ou cuidador responsável. Não foi possível localizar outros dois longevos. Assim, participaram da pesquisa 11 longevos.

Com a determinação dos participantes, realizou-se contato inicial por telefone, quando se explicou a finalidade do estudo ao longevo ou ao seu responsável - a depender da escolha dos participantes, visto que alguns idosos preferiram que a explicação fosse efetuada a seus cuidadores. Havendo concordância na participação, era marcada a visita domiciliar. As visitas ocorreram entre dezembro de 2017 e fevereiro de 2018, sendo realizada em sete municípios circunvizinhos ao do serviço de saúde onde a UTI estava localizada. Não houve recusas em nenhuma das etapas da pesquisa.

Para a coleta de dados, utilizou-se a entrevista aberta, com oito questões norteadoras, elaboradas previamente, e questões fechadas relacionadas aos aspectos sociodemográficos dos participantes. As respostas foram registradas por meio de um gravador. Utilizaram-se, ainda, formulários para avaliação do Índice de Katz e de Lawton de cada longevo, visando avaliar a sua capacidade para realizar Atividades Básicas de Vida Diária (ABVD) e Atividades Instrumentais de Vida Diária (AIVD), respectivamente, além de um roteiro para observação da estrutura do domicílio, do Ministério da Saúde ${ }^{9}$.

A fim de preservar a identidade dos participantes, foram empregados letras e números para diferenciar os depoimentos. Utilizou-se a letra "L" de longevo, seguida de "M" ou "F" para designar sexo masculino ou feminino, e por algarismo numérico apresentando a ordem em que as entrevistas foram realizadas; por exemplo LM1, LM2, LF3... até LM11.

\section{Análise dos depoimentos}

Os dados foram analisados aplicando-se a técnica de Análise de Conteúdo Temática, proposta por Laurence Bardin, que usa procedimentos sistemáticos e objetivos de descrição do conteúdo das mensagens por meio de três etapas: 1) préanálise;2) exploração do material; e 3 ) tratamento dos resultados e interpretação ${ }^{10}$.

Na pré-análise, construiu-se o corpus da pesquisa ${ }^{11}$, por meio da leitura flutuante, com a identificação e seleção das ideias relevantes obtidas na coleta de dados, e foco no objeto do estudo. Na etapa de exploração, houve o aprofundamento do estudo mediante a leitura exaustiva dos depoimentos, com o intuito de categorizar os resultados conforme os temas identificados. Por fim, na etapa de tratamento, atentou-se para a interpretação, a homogeneidade, objetividade e produção de resultados que pudessem trazer novos conhecimentos. Nesse momento, foi utilizado também o referencial teórico do Modelo de Adaptação de Callista Roy, para as reflexões dos discursos dos participantes.

\section{Aspectos Éticos}

A pesquisa seguiu as orientações da Resolução n. 0466 , do Conselho Nacional de Saúde. Para tanto, o projeto de trabalho foi encaminhado ao Comitê de Ética em Pesquisa (CEP) da Universidade do Estado da Bahia, obtendo aprovação pelo Certificado de Apresentação para Apreciação Ética n. 73792317.3.0000.0057, em 11 de setembro de 2017.

\section{RESULTADOS}

Participaram 11 longevos residentes em municípios de pequeno e médio porte, dos quais cinco apresentavam estilo de vida rural. A maioria dos participantes era do sexo feminino, casados $(n=05)$, com ensino fundamental incompleto $(n=08)$ e se autodeclaram brancos $(n=05)$. Todos informaram receber renda de até dois salários-mínimos e estarem aposentados. A idade variou entre 80 e 94 anos. No que concerne à hospitalização, 0 tempo de internação na UTI variou de 3 a 39 dias, com predomínio dos distúrbios gastrointestinais, como abdome agudo obstrutivo, coleperitônio e tumor gástrico.

No retorno domiciliar, em relação ao desempenho das ABVD, somente dois longevos foram identificados como independentes para todas as atividades; os demais possuíam dependências em uma, duas, quatro, cinco ou todas as seis funções elencadas. Quanto às AIVD, a maioria $(n=5)$ foi classificada como dependente grave, dois como totalmente dependentes, e somente um apresentou dependência leve; nenhum longevo foi avaliado como independente para essa classificação. O Quadro 1 apresenta as características coletadas dos longevos no momento da internação na UTI e durante a visita em seus domicílios. 
Quadro 1. Aspectos sociodemográficos dos longevos e características referentes a idade, quantidade de dias de internação na Unidade de Terapia Intensiva, diagnóstico no momento da internação e resultados da Atividade Básica de Vida Diária e da Atividade Instrumental de Vida Diária após retorno domiciliar. Bahia, 2018.

\begin{tabular}{|c|c|c|c|c|c|}
\hline $\begin{array}{l}\text { Identificação } \\
\text { Escolaridade } \\
\text { Estado civil Raça/ } \\
\text { cor Renda }\end{array}$ & Idade & $\begin{array}{c}\text { Dias de } \\
\text { internação } \\
\text { na UTI }\end{array}$ & Diagnóstico na UTI & Resultado da ABVD & Resultado da AIVD \\
\hline $\begin{array}{l}\text { LM1 Não } \\
\text { alfabetizado Viúvo } \\
\text { Branco Até dois } \\
\text { salários-mínimos }\end{array}$ & 92 & 39 & $\begin{array}{l}\text { Bradiarritmia grave } \\
+ \text { Infarto Agudo } \\
\text { do Miocárdio + } \\
\text { miocardiopatia } \\
\text { dilatada }\end{array}$ & Muito dependente & $\begin{array}{l}\text { Totalmente } \\
\text { dependente }\end{array}$ \\
\hline $\begin{array}{l}\text { LM2 Não } \\
\text { alfabetizado } \\
\text { Solteiro Branco } \\
\text { Até dois salários- } \\
\text { mínimos }\end{array}$ & 88 & 17 & $\begin{array}{l}\text { Pós-operatório } \\
\text { Imediato de } \\
\text { Laparotomia } \\
\text { Exploratória } \\
\text { (hemicolectomia a } \\
\text { Direita) + abdome } \\
\text { agudo obstrutivo + } \\
\text { sepse }\end{array}$ & $\begin{array}{l}\text { Independente para todas } \\
\text { as atividades, e apresenta } \\
\text { incontinência urinária e/ou fecal } \\
\text { ocasionalmente. }\end{array}$ & $\begin{array}{l}\text { Dependência } \\
\text { grave }\end{array}$ \\
\hline $\begin{array}{l}\text { LF3 Ensino } \\
\text { fundamental } \\
\text { incompleto Viúva } \\
\text { Branca Até dois } \\
\text { salários-mínimos }\end{array}$ & 82 & 09 & $\begin{array}{l}\text { Drenagem de } \\
\text { abscesso }\end{array}$ & $\begin{array}{l}\text { Recebe assistência no banho em } \\
\text { mais de uma parte do corpo; recebe } \\
\text { assistência para pegar as roupas ou } \\
\text { para vestir-se; deita-se e levanta-se } \\
\text { da cama ou da cadeira com auxílio; } \\
\text { apresenta perdas urinárias e/ou } \\
\text { fecais ocasionais. Alimenta-se sem } \\
\text { assistência, exceto para cortar carne } \\
\text { ou passar manteiga no pão. }\end{array}$ & $\begin{array}{l}\text { Dependência } \\
\text { grave }\end{array}$ \\
\hline $\begin{array}{l}\text { LF6 Ensino } \\
\text { fundamental } \\
\text { incompleto Viúva } \\
\text { Parda Até dois } \\
\text { salários-mínimos }\end{array}$ & 81 & 03 & $\begin{array}{l}\text { Tromboembolismo } \\
\text { pulmonar? } \\
\text { Cardiopatia + } \\
\text { Hipertensão Arterial } \\
\text { Sistêmica }\end{array}$ & $\begin{array}{l}\text { Independente para todas as } \\
\text { atividades, mas apresenta perdas } \\
\text { urinárias e/ou fecais ocasionais. }\end{array}$ & $\begin{array}{l}\text { Dependência } \\
\text { moderada }\end{array}$ \\
\hline
\end{tabular}

Fonte: Dados da pesquisa 
Quadro 1. Continuação...

\begin{tabular}{|c|c|c|c|c|c|}
\hline $\begin{array}{c}\text { Identificação } \\
\text { Escolaridade } \\
\text { Estado civil Raça/ } \\
\text { cor Renda }\end{array}$ & Idade & $\begin{array}{l}\text { Dias de } \\
\text { internação } \\
\text { na UTI }\end{array}$ & Diagnóstico na UTI & Resultado da ABVD & Resultado da AIVD \\
\hline $\begin{array}{l}\text { LF7 Ensino } \\
\text { fundamental } \\
\text { incompleto Casada } \\
\text { Parda Até dois } \\
\text { salários-mínimos }\end{array}$ & 81 & 16 & $\begin{array}{l}\text { Insuficiência Cardíaca } \\
\text { Congestiva }+ \\
\text { Pneumonia }\end{array}$ & $\begin{array}{l}\text { Dependente para todas as } \\
\text { atividades }\end{array}$ & $\begin{array}{l}\text { Dependência } \\
\text { grave }\end{array}$ \\
\hline $\begin{array}{l}\text { LF8 Ensino } \\
\text { fundamental } \\
\text { incompleto Viúva } \\
\text { afuza Até dois } \\
\text { salários-mínimos }\end{array}$ & 94 & 05 & $\begin{array}{l}\text { Pós-operatório } \\
\text { Imediato de } \\
\text { Laparotomia } \\
\text { Exploratória (tumor } \\
\text { gástrico) + obstipação } \\
\text { + Hipertensão Arterial } \\
\text { Sistêmica + Diabetes } \\
\text { Mellitus }\end{array}$ & $\begin{array}{l}\text { Independente para todas as } \\
\text { atividades }\end{array}$ & Dependência leve \\
\hline $\begin{array}{l}\text { LF9 Ensino } \\
\text { fundamental } \\
\text { incompleto Casada } \\
\text { Branca Até dois } \\
\text { salários-mínimos }\end{array}$ & 84 & 06 & $\begin{array}{l}\text { Hipertensão } \\
\text { Arterial Sistêmica + } \\
\text { hematoma cerebral + } \\
\text { plaquetopenia }\end{array}$ & $\begin{array}{l}\text { Independente para todas as } \\
\text { atividades, mas apresenta perdas } \\
\text { urinárias e/ou fecais ocasionais. }\end{array}$ & $\begin{array}{l}\text { Dependência } \\
\text { moderada }\end{array}$ \\
\hline $\begin{array}{l}\text { LM10 Ensino } \\
\text { fundamental } \\
\text { incompleto } \\
\text { Casado Pardo } \\
\text { Até dois salários- } \\
\text { mínimos }\end{array}$ & 85 & 03 & $\begin{array}{l}\text { Pós-operatório } \\
\text { Imediato de correção } \\
\text { de fratura de fêmur } \\
\text { + trauma com fratura } \\
\text { de úmero + fêmur }\end{array}$ & $\begin{array}{l}\text { Independente para todas as } \\
\text { atividades, menos banho, vestir-se, } \\
\text { ir ao banheiro e para cortar a carne. }\end{array}$ & $\begin{array}{l}\text { Dependência } \\
\text { grave }\end{array}$ \\
\hline $\begin{array}{l}\text { LM11 Não } \\
\text { alfabetizado União } \\
\text { estável Preto Até } \\
\text { dois salários - } \\
\text { mínimos }\end{array}$ & 80 & 07 & Abdome Agudo & $\begin{array}{l}\text { Independente para todas as } \\
\text { atividades. }\end{array}$ & $\begin{array}{l}\text { Dependência } \\
\text { moderada. }\end{array}$ \\
\hline
\end{tabular}

Fonte: Dados da pesquisa

Em relação aos aspectos estruturais das residências visitadas, observou-se que as casas não eram adaptadas para as atividades de cuidados diários.

A análise e interpretação dos discursos, à luz da Teoria da Adaptação de Callista Roy, permitiram o delineamento de duas categorias temáticas: Alterações do padrão de resposta às necessidades fisiológicas e perda de autonomia e Alterações psicossociais e comprometimento na realização de atividades cotidianas de lazer e laborais.

\section{Alterações do padrão de resposta às necessidades fisiológicas e perda de autonomia}

O modo fisiológico da Teoria da Adaptação de Roy corresponde à forma como a pessoa responde aos estímulos internos e externos, cujas respostas são comportamentos originados a partir desses estímulos, por intermédio das manifestações fisiológicas apresentadas pelo organismo ${ }^{12}$. As manifestações fisiológicas presentes nos comportamentos dos longevos do estudo foram identificadas nos discursos abaixo:

Agora eu enfastio, não como quase nada [...] gostava mais de suco (LF6).

Não estou me alimentando quase nada, um dia eu como, no outro não. Por isso estou sentindo muita preocupação (LM11).

Não uso mais fralda [...]. Toda hora tenho que ir ao banheiro. Têm horas que não dá tempo nem de chegar ao banheiro (LF3). 
Levanto cinco a seis vezes na noite para poder urinar e sai pouquinha. E quanto às fezes, eu já fui ao banheiro hoje umas duas ou três vezes e não sai (LM4).

Eu cheguei (do hospital), fiquei acamada, a mente estava boa. Mas não tinha vontade de me alimentar e nem de dormir [...] E tomando medicamento diário (LF9).

As funções fisiológicas são essenciais para garantir condições e qualidade de vida ao longevo, e as alterações desencadeadas aumentam a possibilidade de dependência e a necessidade de cuidador, o que pode levar à perda de autonomia para a realização das ABVD e AIVD. Essas respostas provocam alterações importantes na vida do longevo, como abordado a seguir:

Eu tomo banho sentado, quem me dá banho é $X,[\ldots]$ porque as pernas não aguentam (LM1).

De poucos dias para cá, eu estou andando com isso aqui (andador), antes eu não estava nem andando. Para sair, os meninos me seguram de lado, mas agora eu estou tentado a andar [...] (LM4).

Fiquei mais de um mês em repouso. Saía dali, sentava aqui. Eu fiquei vários dias sem andar direito, sentia peso nas pernas. Ficava mais acamada (LF6).

Eu não faço nada, fico deitada na cama [...], só ando na cadeira de rodas. Para tomar banho, me pegam e me levam (LF7).

Aqui a dificuldade (para adaptar) era só quando eu queria ir ao banheiro ou no quintal (LM4).

Eu fico aqui, têm horas que eu me sento, têm horas que ela (filha) me coloca no sofá, [...] tomo um sol tambéme depois me coloca aqui. Falar a verdade, eu vivo mais é deitada. O dia que chove eu não durmo, sinto muita dor nos ossos (LF5).

Aqui, no banheiro e no quarto, elas (filhas) fizeram barras para eu pegar. Elas me davam banho sentada, agora eu já tomo em pé, mesmo assim, eu não me abaixo para lavar os pés, nem as costas, nem a cabeça. Preciso de muita ajuda ainda. Eu senti dificuldade para andar, sentar, levantar e ir ao banheiro. Eles tinham que me levar ou eu fazia o xixi na aparadeira (LF3).

Alguns longevos apontaram também o desconforto vivenciado pela necessidade de uso de fraldas geriátricas, ainda que temporariamente, conforme elencado nas falas a seguir:

De toda essa doença minha, o que eu tive mais dificuldade foi usar fralda. Usar fralda para os outros cuidarem é ruim demais (LM10).

De quando eu saí da UTI, nos primeiros dias, ela (companheira) me ajudava, a levantar. Nos primeiros dias precisei usar fralda (LM11).

\section{Alterações psicossociais e comprometimento na realização de atividades cotidianas de lazer e laborais}

Por meio dos depoimentos, os longevos retrataram sentimento de tristeza e insatisfação devido às dificuldades, privações e/ ou impossibilidade de voltar a realizar atividades de lazer, após o acometimento da doença crítica. Logo, foi possível observar as modificações que os agravos geraram nos comportamentos dessas pessoas de 80 anos ou mais, e que culminaram em mudanças significativas no seu estilo de vida.

Eu cuidava da minha roça, fazia as minhas viagens, minhas pescarias, eu fazia de tudo dentro de casa. Agora, depois que eu fiquei doente, o negócio desandou (LM2).

Gosto muito de dançar [...]. Me faria feliz se eu dançasse, mas eu não posso [...]. Sou católico, [...] o padre faz festa e traz sanfoneiro [...]. Agora eu quero ver se terá a festa depois que eu melhorar (LM1).

Eu tenho saudade de fazer tudo, pois, para quem era acostumada, é duro [...]. Eu fazia tudo e não sentia (LF3).

Há de se considerar que, a despeito dos problemas adquiridos a partir do adoecimento, o longevo pode apresentar fragilidades e vulnerabilidades, advindas da própria idade e do seu processo de envelhecimento, que dificultam a realização de atividades de lazer. Contudo, os próprios longevos destacaram justamente a doença como marco para o comprometimento do seu estilo de vida.

Neste estudo, além das mudanças percebidas referentes às atividades de lazer, os depoentes salientaram o afastamento das suas atividades laborais, que também culminou com modificações em seu estilo de vida.

Não posso cuidar de nada, não posso trabalhar na roça. Então fico preocupado, porque eu não posso fazer nada das coisas que eu gostava de fazer e ter (LM11).

Lá na roça, até hoje, eu tenho uma criação de bois que eu tenho que olhar. Agora são os meninos que vão lá ver. Eu falo com eles: Deus me livre de eu morrer e vocês não darem continuidade, tem que estar olhando a roça, a cerca com o vizinho [...]. E esses meninos aí não fazem o que eu faço na roça. E agora eu não tenho confiança de ir. Porque, às vezes, eu andava lá por aqueles matos olhando se estava tudo direito. E agora, com isso aí, eu não vou sozinho (LM4).

Tudo dentro de casa eu fazia [...]. Hoje, posso fazer assim, alguma comidinha. Lavar a roupa não é possível, porque eu tenho medo de cair e as mãos também estão meio travadas (LF3).

Tais respostas comportamentais de adaptação ineficaz culminaram em fragilidades, sentimentos de tristeza e incapacidade, que desencadearam desesperança em relação à vida, conforme 
observado na fala de LM1, quando ele diz: Aqui, eu não faço nada [...] eu estou quase morto, minha filha.

\section{DISCUSSÃO}

Sobre o tempo de internação na UTI, o estudo em tela apresenta resultados semelhantes a uma pesquisa realizada com 128 longevos, internados na UTI do hospital de ensino da Universidade Federal de São Paulo, em que o período de internação variou entre 3 e 42 dias; hipertensão e diabetes foram as comorbidades mais frequentes. Também houve predomínio de pessoas idosas do sexo feminino, que se autodeclaram de cor branca, aposentadas, não letradas ou com ensino fundamental incompleto, e que recebiam entre um e dois salários-mínimos ${ }^{4}$.

Um estudo realizado na Inglaterra, País de Gales e Irlanda do Norte, cujo objetivo era explorar as admissões, uso de recursos e o risco de mortalidade hospitalar em pacientes mais velhos, internados entre 1997 e 2016, em UTI gerais para adultos, evidenciou aumento no número de hospitalizações de pessoas idosas, sendo que esse aumento foi proporcionalmente maior do que a expansão de idosos na população em geral no Reino Unido. Observou-se, também, que os pacientes mais velhos permaneceram mais tempo no hospital após a alta da UTI, e que a taxa de mortalidade hospitalar aumentava com a idade ${ }^{13}$.

O diagnóstico médico que predominou neste estudo em tela está em desacordo com o de outra pesquisa realizada em uma UTI em Salvador (BA), o qual identificou o perfil clínico e sociodemográfico de 252 longevos internados na unidade, e evidenciou que as doenças de causas não infecciosas, com destaque para o Acidente Vascular Cerebral (AVC) e Infarto Agudo do Miocárdio (IAM), foram as principais causas de internação observadas nessa população, representando 84,5\% dos participantes ${ }^{14}$.

A partir das falas dos participantes, é possível verificar que, das cinco necessidades básicas relacionadas à integridade fisiológica descritas na Teoria da Adaptação de Callista Roy-de oxigenação, nutrição, eliminação, atividade e repouso e proteção -, apenas a necessidade relativa à proteção - que se refere à integridade da pele - não foi mencionada pelos longevos. Todas as demais foram relatadas como um problema identificado por eles, após o retorno domiciliar.

Cabe destacar que os problemas de adaptação referentes à oxigenação não podem ser averiguados com exatidão, uma vez que o LM1 refere apenas diminuição do "fôlego", sendo necessária uma avaliação mais detalhada. Quanto à nutrição, observam-se problemas que podem ser alusivos à nutrição, menor do que os requisitos do corpo, e estratégias de resistência ineficazes para os meios de digestão alterados.

Pesquisa realizada em um hospital do Rio Grande do Sul avaliou os fatores que mais dificultavam a alimentação via oral em idosos hospitalizados. A amostra foi constituída por 111 idosos, e os resultados apontaram que os fatores ambientais foram os mais relatados pela população, seguidos de fatores alimentares, fisiológicos e relacionados à equipe ${ }^{15}$.
Os participantes deste estudo, mesmo estando em seus domicílios sob os cuidados de familiares, alegaram dificuldades na aceitação da dieta após a alta hospitalar. Tal fator deveria ser investigado ainda durante a internação, como forma de buscar soluções para o problema. Considerando a importância de manter uma dieta oral adequada e equilibrada a fim de promover uma recuperação mais eficaz, estratégia importante para auxiliar a pessoa de 80 anos ou mais nesse quesito seria receber orientações da nutrição no momento da alta e, preferencialmente, realizar acompanhamento com o profissional também após o retorno domiciliar, visando melhor aceitação da dieta e promoção do equilíbrio das necessidades nutricionais.

Quanto aos problemas relacionados à eliminação, percebese que os de adaptação são provocados devido à incontinência urinária e obstipação. A incontinência urinária é percebida como uma das mais importantes síndromes geriátricas e deve ser valorizada na prática clínica ${ }^{16}$.

A hospitalização favorece o desenvolvimento de incontinências nos longevos, em virtude da condição de acamado e do uso de fraldas, e essa é uma importante questão a ser pensada durante a hospitalização e no preparo para a alta da unidade.

Tal afirmativa é evidenciada em um estudo que objetivou obter a incidência do desenvolvimento de incontinência, no momento da alta hospitalar, em pacientes com idade igual ou superior a 65 anos, diagnosticados com demência ou deficiência cognitiva, e que eram continentes pré-admissão. Os resultados apontaram que, dos 100 idosos avaliados, 57\% apresentaram alguma incontinência durante a hospitalização, sendo 36\% incontinência urinária; destes, $2 \%$ também apresentaram incontinência fecal no momento da alta ${ }^{17}$.

Estudo realizado em Montes Claros (MG), com 686 idosos - em sua maioria do sexo feminino -, identificou prevalência da incontinência urinária em $31,1 \%$ delas, e em $23,2 \%$ nos idosos do sexo masculino. Em ambos os sexos, a fragilidade foi um fator que esteve associado à presença da incontinência urinária ${ }^{16}$.

Referente às atividades e repouso, observaram-se, na fala reportada pela LF9, problemas de adaptação após a alta hospitalar, que podiam ter sido oriundos da condição de estar acamada, proporcionando uma mobilidade restrita e um padrão inadequado de atividade e repouso, com privação do sono.

Pesquisa realizada em um hospital universitário -cujo objetivo foi analisar a prevalência e fatores associados à necessidade de auxílio para realizar as ABVD no pós-alta hospitalar de idosos-, identificou que, dos 144 avaliados, 43,1\% necessitaram de auxílio para realizar ABVD após a alta hospitalar. Os pesquisadores destacaram a necessidade de se ter uma relação efetiva entre o atendimento hospitalar e continuidade dos cuidados no retorno domiciliar, e enfatizaram que, quando tais cuidados não são adequados, resultam em reinternações, surgimento de novas doenças ou perda da autonomia do idoso' ${ }^{1}$.

No estudo em tela, a maioria dos longevos permaneceu internada na UTI por um período considerável, de seis a dez dias, fato que pode ter interferido no aumento da necessidade de cuidados e dependência funcional. 
Estudo que avaliou 373 idosos cadastrados em uma Estratégia de Saúde da Família (ESF), do município de Taiboeiras (MG), observou que as variáveis que apresentavam maior associação com o estado funcional de dependência entre eles era viver sem um companheiro, não ser alfabetizado e ser do sexo feminino. Relacionado à idade, as variáveis foram categorizadas em idade: $\geq 80$ anos e $<80$ anos, demonstrando que ter menos de 80 anos relacionava-se a maior independência para as ABVDs ${ }^{18}$.

Em relação às variáveis, as que tiveram resultados divergentes a esta pesquisa foram as relacionadas ao estado civil e à alfabetização, uma vez que a maioria era casada e possuía o ensino fundamental incompleto. Ainda assim, percebe-se que a variável idade pode estar relacionada a maior dependência da capacidade funcional, quando comparada a outras faixas etárias, visto que grande parte dos longevos apresentava dependências em uma ou mais funções para a realização da ABVD, e eram dependentes graves ou totalmente dependentes nas AIVD.

A perda da funcionalidade da pessoa idosa é o condicionante de maior relevância para desfechos desfavoráveis nessa população, posto que implica diminuição da qualidade de vida da pessoa, de seus familiares e cuidadores. Como consequência da diminuição da capacidade funcional, tem-se o aumento de hospitalização, institucionalização e mortalidade do idoso ${ }^{19}$.

Dessa forma, é importante que o idoso seja estimulado pelos profissionais de saúde, familiares e seu cuidador, na realização das ABVD e AIVD durante a hospitalização, sempre que possivel, e em seu domicílio, de forma segura, para promoção da independência e autonomia ${ }^{20}$. Essas ações fornecerão mecanismos porque o longevo apresente respostas comportamentais favoráveis para proporcionar uma vida mais ativa e funcional, devendo ser iniciadas ainda durante o internamento.

A presença de alterações psicossociais vivenciadas pelo longevo, no retorno domiciliar, após hospitalização, contribui para uma resposta comportamental eficaz ou não, dificultando a sua recuperação e reabilitação. Nesse sentido, o objetivo é promover meios que proporcionem a conservação ou a recuperação da saúde, mediante intervenções necessárias para essa finalidade ${ }^{21}$.

Pesquisa realizada com 6.913 idosos de 60 anos ou mais, residentes em áreas urbanas de 59 municípios do Rio Grande do Sul, identificou que $22,5 \%$ dos longevos e $9,2 \%$ dos idosos mais jovens alegaram não sair de suas casas semanalmente, demonstrando que o percentual de longevos que não saíam era significativamente maior. Em ambos os grupos, a falta de segurança, seguida pela dificuldade de locomoção, foram os relatos mais frequentes. Os resultados obtidos sugerem que os longevos exibiram maior limitação para saírem de casa, quando comparados aos idosos mais jovens ${ }^{22}$.

Ademais, o comportamento de sedentarismo em idosos é um fator importante na identificação de suspeita de transtornos mentais comuns nessa população, configurando-se um fator de risco que deve ser investigado e valorizado por profissionais de saúde ${ }^{23}$.

Nesta pesquisa, foi possível observar depoimentos que revelaram um comportamento ineficaz, demonstrando a necessidade de se provocar mudanças no ambiente, tornando-o adaptável, e promover a saúde e a qualidade de vida das pessoas de 80 anos ou mais. Nota-se que grande parte apresentou modificações no seu estilo de vida, as quais impactaram negativamente na adaptação após o retorno domiciliar, ocorrendo privações relacionadas às atividades de lazer, que eram rotineiras antes da hospitalização.

No que concerne ao idoso hospitalizado, pesquisa realizada no México, cujo objetivo foi avaliar os escores de depressão e outras variáveis de saúde física e mental em idosos com e sem depressão, internados em uma enfermaria de traumatologia por fratura de quadril, evidenciou alta frequência de depressão, especialmente em mulheres e indivíduos com mais de 81 anos de idade ${ }^{24}$.

Outro estudo, realizado com 552 idosos hospitalizados, apontou que havia maior prevalência para depressão nas pessoas idosas do sexo feminino, solteiras, com menor renda familiar, fumantes e que tinham passado pela internação hospitalar nos últimos 12 meses anteriores à entrevista. Os resultados demonstraram, ainda, que a participação regular em atividades de lazer reduzia significativamente o risco de desenvolver a depressão, e que essa proteção era de $62 \%$ para aqueles que participaram de pelo menos três atividades de lazer no último mês ${ }^{25}$.

A participação do idoso em atividades de lazer - como passeios, viagens e danças - é de extrema importância para estimular a sua integração social e favorecer a ampliação da rede de apoio, da comunicação e na autoestima, acarretando benefícios psicológicos e sociais ${ }^{25}$.

Neste estudo, foi possível perceber que, em alguns longevos, o adoecimento e a hospitalização desencadearam mudanças que impossibilitaram a realização de atividades laborais anteriormente desenvolvidas, gerando preocupação, insegurança e angústia. Tal constatação foi principalmente percebida naqueles que desempenhavam atividades laborais na zona rural, visto que tais atividades exigem força e trabalho braçal, cuja realização pode ser impossibilitada temporária ou permanentemente por pessoas de 80 anos ou mais pós-alta hospitalar.

É importante ressaltar que, devido à hospitalização, alguns longevos apresentaram diminuição da sua capacidade funcional. Em razão disso, demonstravam descrenças em vislumbrar seu retorno ao trabalho, e esse sentimento gerou tristeza, uma vez que essas atividades haviam sido realizadas por eles durante toda uma vida.

Estudo ocorrido com 87 longevos identificou que 60,92\% destes ainda exerciam atividades laborais nas casas em que residiam ou em serviços voluntários, mesmo estando aposentados. Os resultados demonstraram que $6,89 \%$ dos longevos eram os responsáveis diretos ou indiretos nos cuidados de familiares; $42,52 \%$ trabalhavam em hortas, jardins ou com animais domésticos e $23,43 \%$ deles exerciam atividades domésticas ${ }^{26}$.

Observa-se que, na ausência da doença, muitos longevos dispõem de uma vida ativa e desenvolvem atividades laborais, seja no campo ou no lar. O acometimento da doença e a necessidade de cuidados intensivos em uma unidade fechada podem determinar transformações que comprometerão a realização de atividades cotidianas, além da possibilidade de tornar sedentário quem antes nesse processo era ativo. 


\section{CONCLUSÕES E IMPLICAÇÕES PARA A PRÁTICA}

Como respostas comportamentais que contribuem para uma adaptação ineficaz, foram identificadas aquelas relacionadas aos modos fisiológicos e psicossociais. Ao que se refere às manifestações fisiológicas, foi possível perceber que elas aumentam a possibilidade de dependência e perda da autonomia do longevo para a realização das ABVD e AIVD, levando à necessidade de um cuidador. Essa relação pode estar associada à condição de hospitalização prévia em uma UTI, e desencadear fragilidades e perda da funcionalidade, ainda mais ao se considerar o fator idade.

As alterações psicossociais percebidas estavam relacionadas com a dificuldade de realização de atividades cotidianas de lazer e laborais. Foi retratado que, após o acometimento da doença crítica que culminou com essas alterações, os longevos apresentavam sentimentos de tristeza e insatisfação em virtude das dificuldades e privações adquiridas, implicando mudanças significativas em seu estilo de vida.

Perceberam-se dificuldades vivenciadas pelo longevo no retorno domiciliar, após internação na UTI, que provocaram alterações significativas na realização de atividades cotidianas, evidenciando a necessidade de se iniciar o preparo para o retorno domiciliar, pela equipe multiprofissional, ainda durante a hospitalização.

Evidencia-se uma interdependência das dimensões biopsicossociais em todas as categorias, sendo possível visualizar que uma interfere na outra. Tal fato condiz com a visão da Teoria da Adaptação de Roy, que reconhece a pessoa como um sistema holístico e adaptável.

Mediante a realização desta pesquisa, foi possível perceber o quanto os longevos se sentem gratos e valorizados com a visita do profissional da saúde que busca informações referentes às suas condições de saúde, sendo essa uma importante ferramenta para o cuidado da pessoa idosa.

Como limitação do estudo, verificou-se a impossibilidade de se investigar as ABVD e AIVD realizadas pelos longevos participantes desta pesquisa, anteriormente à hospitalização, visto que as escalas só foram aplicadas após a alta hospitalar. Dessa forma, não foi possível estabelecer uma comparação da capacidade funcional do longevo antes e após a hospitalização.

\section{AGRADECIMENTOS}

À Universidade Federal da Bahia (UFBA) e à Universidade do Estado da Bahia (UNEB).

\section{FINANCIAMENTO}

O presente trabalho foi realizado com apoio da Coordenação de Aperfeiçoamento de Pessoal de Nível Superior - Brasil (CAPES) - Código de Financiamento 001, e da Fundação de Amparo à Pesquisa do Estado da Bahia (FAPESB).

\section{CONTRIBUIÇÕES DOS AUTORES}

Desenho do estudo. Jessica Lane Pereira Santos. Larissa Chaves Pedreira. Juliana Bezerra do Amaral. Larissa Simões da Cruz Pessoa.

Aquisição de dados. Jessica Lane Pereira Santos. Larissa Chaves Pedreira. Juliana Bezerra do Amaral.

Análise de dados e interpretação dos resultados. Jessica Lane Pereira Santos. Larissa Chaves Pedreira. Juliana Bezerra do Amaral. Larissa Simões da Cruz Pessoa. Elaine de Oliveira Souza. Aline Cristiane de Sousa Azevedo Aguiar

Redação e revisão crítica do manuscrito. Jessica Lane Pereira Santos. Larissa Chaves Pedreira. Juliana Bezerra do Amaral. Larissa Simões da Cruz Pessoa. Elaine de Oliveira Souza. Aline Cristiane de Sousa Azevedo Aguiar

Aprovação da versão final do artigo. Jessica Lane Pereira Santos. Larissa Chaves Pedreira. Juliana Bezerra do Amaral. Larissa Simões da Cruz Pessoa. Elaine de Oliveira Souza. Aline Cristiane de Sousa Azevedo Aguiar

Responsabilidade por todos os aspectos do conteúdo e a integridade do artigo publicado. Jessica Lane Pereira Santos. Larissa Chaves Pedreira. Juliana Bezerra do Amaral. Larissa Simões da Cruz Pessoa. Elaine de Oliveira Souza. Aline Cristiane de Sousa Azevedo Aguiar.

\section{EDITOR ASSOCIADO}

Cristina Rosa Soares Lavareda Baixinho (1)

\section{EDITOR CIENTÍFICO}

Marcelle Miranda da Silva (D)

\section{REFERÊNCIAS}

1. Bordin D, BlanskiGrden CR, Andreani Cabral LP, Krum EA, Savin Sanson I. Auxílio à realização de atividades básicas de vida diária no pós-alta hospitalar de idosos. RevSALUS. 2021 jan;2(2):38-44. https:// doi.org/10.51126/revsalus.v2i2.59.

2. Rodriguez AH, Bub MBC, Perão OF, Zandonadi G, Rodriguez MJH Características epidemiológicas e causas de óbitos em pacientes internados em terapia intensiva. Rev Bras Enferm. 2016 abr;69(2):22934. http://dx.doi.org/10.1590/0034-7167.2016690204i.

3. Couto FBD. Cuidando do idoso no hospital e em internação domiciliar: 0 que há de diferente? Rev Kairós Gerontologia. 2015;18(N. esp. 19):5776. https://doi.org/10.23925/2176-901X.2015v18iEspecial18p57-76.

4. Billett MC, Campanharo CRV, Lopes MCBT, Batista REA, Belasco AGS, Okuno MFP. Functional capacity and quality of life of hospitalized octogenarians. Rev Bras Enferm. 2019;72(Suppl 2):43-8. http://dx. doi. org/10.1590/0034-7167-2017-0781.

5. Gerth AMJ, Hatch RA, Young JD, Watkinson PJ. Changes in health-related quality of life after discharge from an intensive care unit: a systematic review. Anaesthesia. 2019;74(1):100-8. http://dx.doi.org/10.1111/ anae.14444.

6. Santos JLP, Pedreira LC, Amaral JB, Silva VA, Pereira A, Aguiar ACSA. Adaptação de Longevos no Domicílio após Internação na Unidade de Terapia Intensiva e Alta Hospitalar. Texto Contexto Enferm. 2019;28:e20180286. http://dx.doi.org/10.1590/1980-265x-tce-2018-0286.

7. Monteiro AKC, Costa CPV, Campos MOB, Monteiro AKC. Aplicabilidade da Teoria de Callista Roy no Cuidado de Enfermagem ao Estomizado. 
Rev Enferm Atenção Saúde. 2016 jan/jul;5(1):84-92. http://dx.doi. org/10.18554/reas.v5i1.1625.

8. Roy C, Andrews HA. Teoria da Enfermagem - O Modelo de Adaptação de Roy. Lisboa: Instituto Piaget; 2001.

9. Ministério da Saúde (BR). Secretaria de Atenção à Saúde. Departamento de Atenção Básica. Envelhecimento e saúde da pessoa idosa [Internet]. Brasília: Ministério da Saúde; 2006 [citado 2018 Mar 20]. Disponível em:http://189.28.128.100/dab/docs/publicacoes/cadernos_ab/abcad19. pdf[

10. Bardin L. Análise de conteúdo. 5aㅡ ed. Lisboa (PT): Edições 70; 2011.

11. Mendes RM, Miskulin RGS. A análise de conteúdo como uma metodologia. Cad Pesq. 2017 set;47(165):1044-66. http://dx.doi. org/10.1590/198053143988.

12. Coelho SMS; Mendes IMDM. Da pesquisa à prática de enfermagem aplicando o Modelo de Adaptação de Roy. Esc Anna Nery (impr.) 2011;15(4):845-50. http://dx.doi.org/10.1590/S1414-81452011000400026.

13. Jones A, Toft-Petersen AP, Shankar-Hari M, Harrison DA, Rowan KM. Demographic shifts, case mix, activity, and outcome for elderly patients admitted to adult general ICUs in England, Wales, and Northern Ireland. Crit Care Med. 2020;48(4):466-74. http://dx.doi.org/10.1097/ CCM.0000000000004211.

14. Silva JBVB, Pedreira LC, Santos JLP, Barros CSMA, David RAR. Perfil clínico de longevos em uma unidade de terapia intensiva. Acta Paul Enferm. 2018 fev;31(1):39-45. http://dx.doi.org/10.1590/19820194201800007.

15. Lima DF, Pelzer MT, Barros EJL, Semedo DSRC, Rosales RA. Fatores que dificultam a alimentação por via oral do idoso hospitalizado. Enferm glob. 2017;16(48):429-64. http://dx.doi.org/10.6018/eglobal.16.4.271841.

16. Carneiro JA, Ramos GCF, Barbosa ATF, Medeiros SM, Lima CA, Costa $\mathrm{FM}$ et al. Prevalência e fatores associados à incontinência urinária em idosos não institucionalizados. Cad Saude Colet. 2017;25(3):268-77. http://dx.doi.org/10.1590/1414-462x201700030295.

17. Furlanetto K, Emond K. "Will I come home incontinent?" Aretrospective file review: Incidence ofdevelopment of incontinence andcorrelation with length of stay in acutesettings for people with dementia orcognitive impairment aged 65 years andover. Collegian. 2016;23(1):79-86. http:// dx.doi.org/10.1016/j.colegn.2014.09.013.
18. Silva CSO, Barbosa MMS, Pinho L, Figueiredo MFS, Amaral CO, Cunha FO et al. Estratégia saúde da família: relevância para a capacidade funcional de idosos. Rev Bras Enferm. 2018;71(Supl 2):740-6. http:// dx.doi.org/10.1590/0034-7167-2017-0078.

19. Ministério da Saúde (MS). Secretaria de Atenção à Saúde. Departamento de Atenção Especializada e Temática - DAET. Coordenação Saúde da Pessoa Idosa-COSAPI. Diretrizes para o Cuidado das Pessoas Idosas no SUS: Proposta de Modelo de Atenção Integral. XXX Congresso Nacional de Secretarias Municipais de Saúde [Internet]. Brasília: Ministério da Saúde; 2014 [citado 2018 jun 10]. Disponível em: http://bvsms.saude. gov.br/bvs/publicacoes/diretrizes_cuidado_pessoa_idosa_sus.pdf

20. Llano PMP, Lange C, Nunes DP, Pastore CA, Pinto AH, Casagranda LP. Fragilidade em idosos da zona rural: proposta de algoritmo de cuidados. Acta Paul Enferm. 2017;30(5):520-30. http://dx.doi.org/10.1590/19820194201700075.

21. Costa IKF, Nóbrega WG, Costa IKF, Torres GV, Lira ALBC, Tourinho FSV et al. Pessoas com úlceras venosas: estudo do modo psicossocial do modelo adaptativo de Roy. Rev Gaúcha Enferm (Online). 2011 set;32(3):561-8. http://dx.doi.org/10.1590/S1983-14472011000300018.

22. Navarro JHN, Andrade FP, Paiva TS, Silva DO, Gessinger CF, Bós AJG. Percepção dos idosos jovens e longevos gaúchos quanto aos espaços públicos em que vivem. Ciênc Saúde Coletiva. 2015 fev;20(2):461-70. http://dx.doi.org/10.1590/1413-81232015202.03712014.

23. Silva PAS, Rocha SV, Vasconcelos LRC, Santos CA. Comportamento sedentário como discriminador dos transtornos mentais comuns em idosos. J Bras Psiquiatr. 2017 out;66(4):183-8. http://dx.doi. org/10.1590/0047-2085000000169.

24. Charles-Lozoya S, Cobos-Aguilar H, Barba-Gutiérrez E, Brizuela-Ventura JM, Chávez-Valenzuela S, García-Hernández A et al. Depresión y Evaluación geriátrica en adultos mayores ingresados por fractura de cadera. Rev Med Chile. 2019 ago;147(8):1005-12. http://dx.doi. org/10.4067/S0034-98872019000801005.

25. Gullich I, Duro SMS, Cesar JA. Depressão entre idosos: um estudo de base populacional no Sul do Brasil. Rev Bras Epidemiol. 2016 dez;19(4):691-701. http://dx.doi.org/10.1590/1980-5497201600040001.

26. Farias RG, Santos SMA. Influência dos determinantes do envelhecimento ativo entre idosos mais idosos. Texto Contexto Enferm. 2012 mar;21(1):16776. http://dx.doi.org/10.1590/S0104-07072012000100019. 